\title{
Surrogate Nuclear Reactions using STARS
}

L. A. Bernstein, J. T. Burke, J. A. Church, L. Ahle, J. R. Cooper, R. D. Hoffman, K. Moody, J. Punyon, A. Schiller, E. Algin, C. Plettner, H. Ai, C. W. Beausang, R. F. Casten, R. Hughes, E. Ricard-McCutchan, D. Meyer, J. J. Ressler, J. A. Caggiano, N. V. Zamfir, H. Amro, A. Heinz, P. Fallon, M. A. McMahan, A. O. Macchiavelli, L. W. Phair

\section{January 28, 2005}

International Conference on Nuclear Data for Science and Technology - 2004 Santa Fe, NM, United States September 26, 2004 through October 1, 2004 
This document was prepared as an account of work sponsored by an agency of the United States government. Neither the United States government nor Lawrence Livermore National Security, LLC, nor any of their employees makes any warranty, expressed or implied, or assumes any legal liability or responsibility for the accuracy, completeness, or usefulness of any information, apparatus, product, or process disclosed, or represents that its use would not infringe privately owned rights. Reference herein to any specific commercial product, process, or service by trade name, trademark, manufacturer, or otherwise does not necessarily constitute or imply its endorsement, recommendation, or favoring by the United States government or Lawrence Livermore National Security, LLC. The views and opinions of authors expressed herein do not necessarily state or reflect those of the United States government or Lawrence Livermore National Security, LLC, and shall not be used for advertising or product endorsement purposes. 


\title{
Surrogate Nuclear Reactions using STARS
}

\author{
L.A.Bernstein, J.T.Burke, J.A.Church, L.Ahle, J.R.Cooper, R.D. Hoffman, \\ K. Moody, J. Punyon ${ }^{1}$, A.Schiller ${ }^{2}$, E. Algin ${ }^{3}$ \\ Lawrence Livermore, National Laboratory, Livermore, CA 94551
}

\section{C.Plettner, H. Ai, C.W. Beausang ${ }^{4}$, R.F. Casten, R.Hughes, E. Ricard-McCutchan, D. Meyer, J.J.Ressler, J.A.Caggiano, N.V. Zamfir, H. Amro, A. Heinz A.W. Wright Nuclear Structure Laboratory, Yale University New Haven, CT 06520}

\author{
P.Fallon, M.A. McMahan, A.O. Macchiavelli, L.W. Phair \\ E.O. Lawrence Berkeley National Laboratory Berkeley, CA 94720
}

\begin{abstract}
The results from two surrogate reaction experiments using the STARS (Silicon Telescope Array for Reaction Studies) spectrometer are presented. The surrogate method involves measuring the particle and/or $\gamma$-ray decay probabilities of excited nuclei populated via a direct reaction. These probabilities can then be used to deduce neutroninduced reaction cross sections that lead to the same compound nuclei. In the first experiment STARS coupled to the GAMMASPHERE $\gamma$-ray spectrometer successfully reproduce surrogate $(n, \gamma),\left(n, n^{\prime} \gamma\right)$ and $(n, 2 n \gamma)$ cross sections on ${ }^{155,156} \mathrm{Gd}$ using ${ }^{3} \mathrm{He}$-induced reactions. In the second series of experiments an energetic deuteron beam from the ESTU tandem at the Wright Nuclear Structure Lab at Yale University was used to obtain the ratio of fission probabilities for ${ }^{238} \mathrm{U} /{ }^{236} \mathrm{U}$ and ${ }^{237} \mathrm{U} /{ }^{239} \mathrm{U}$ populated using the ${ }^{236,238} \mathrm{U}(\mathrm{d}, \mathrm{d}$ 'f $)$ and ${ }^{236,238} \mathrm{U}(\mathrm{d}, \mathrm{pf})$ reactions. Results from these experiments are presented and the implications for the surrogate reaction technique are discussed.
\end{abstract}

\section{INTRODUCTION}

Reactions on unstable nuclei are at the core of nucleosynthesis in environments from stars to supernovae to the interior of nuclear weapons. However, the cross sections for many of these reactions are difficult to predict due to the influence of nuclear structure effects and unusual decay modes. Furthermore, these cross sections can be equally difficult to measure due to the short lifetimes and large backgrounds of the radioactive targets involved. The LLNL group is leading an effort to deduce neutroninduced reaction cross sections on unstable nuclei using a technique referred to as the surrogate reaction method. A surrogate reaction experiment involves measuring the decay probabilities of an intermediate nucleus populated using a light-ion induced reaction.
The decay probability is determined through the coincident observation of the ejectile and a "tag" for a specific decay channel. Examples of tags include particles, $\gamma$-rays or fission fragments. The decay probability for channel $\mathrm{x}, P_{x}$, would then be determined through the relation:

$$
P_{X}\left(E_{X}\right)=\frac{N_{X}}{\varepsilon N_{\text {ejectile }}}
$$

Where $\varepsilon$ is the efficiency for detecting the residual nucleus tag, $\mathrm{N}_{\mathrm{x}}$ is the number of residual nuclei tags observed for channel $\mathrm{x}$ and $\mathrm{N}_{\text {ejectile }}$ are the number of coincident ejectiles observed The critical ansatz of the surrogate reaction method is that the intermediate nucleus formed in both the light-ion induced and the

\footnotetext{
${ }^{1}$ Present Address: Department of Physics, The State University of New York at Geneseo, Geneseo NY 14454 USA

${ }^{2}$ Present Address: MSU/NSCL, 1 Cyclotron Rd. East Lansing MI, 48824 USA

${ }^{3}$ Present Addrss: Department of Physics, Osmangazi University, Meselik, Eskisehir, TR-26480.TURKEY.

${ }^{4}$ Present Address: Department of Physics, University of Richmond, Richmod,VA 23173 USA
} 
neutron induced reactions is compound . i.e. its decay probabilities are independent of its formation. Britt and Wilhelmy [1] pioneered the use of the surrogate technique to obtain $(n, f)$ cross sections in the late 1960's and 1970's using light-ion induced direct reactions. Recent work by Younes and Britt [2] has combined these measured probabilities with the fission and optical model calculations to produce the surrogate neutron-induced fission cross section. Younes has also shown [3] that for excitation energies $2.5 \mathrm{MeV}$ or more in excess of the neutron separation energy the decay probabilities become independent of spin (the Weisskopf-Ewing limit) In this limit the relationship between the measured decay probability and the desired neutron-induced reaction cross sections can be written as:

$$
\sigma_{(n, f)}=P\left(E_{X}\right) \bullet \sigma_{\text {reaction }}\left(E_{X}\right)
$$

Where $P\left(E_{x}\right)$ is the measured energy dependent decay probability, and $\sigma_{\text {reaction }}\left(E_{x}\right)$ is the neutron-absorbtion cross section from an optical model calculation.

Two large systematic errors uncertainties dominate surrogate measurements: the determination of $N_{\text {ejectiles }}$ (due to contamination of the particle-singles spectrum from reactions on light contaminants in the target such as Carbon and Oxygen), and the difference in angular momentum of the compound nucleus populated via the light-ion induced and the neutron-induced reactions

The first of the two experiments described in this paper involves the first use of surrogate reactions to probe channels other than fission. The particle-singles issue is bypassed in this experiment by utilizing ratios of $\gamma$-ray decay probabilities rather than the reaction channel probabilities themselves. The second of the experiments circumvents both the particle-singles and the angular momentum mismatch issues by measuring a ratio of fission probabilities for two very similar nuclei $\left({ }^{238} \mathrm{U} /{ }^{236} \mathrm{U}\right.$ and $\left.{ }^{237} \mathrm{U} /{ }^{239} \mathrm{U}\right)$. Although the STARS detector was utilized for both measurements the actual Silicon detectors used differed from one experiment to another. The details of the experiments are discussed in the following sections.

\section{THE STARS+GAMMASPHERE EXPERIMENT}

The first experiment was performed in April 2002 at the 88-Inch cyclotron at Lawrence Berkeley National Laboratory using the GAMMASHERE $\gamma$-ray spectrometer coupled to STARS. In this experiment the SiRi (Silicon RingSTARS. Four panels of SiRi detectors were arrayed in a partial "lampshade configuration designed to provide 8 equally spaced angle ranges between $30^{\circ}$ and $60^{\circ}$. The thickness of the front $\Delta \mathrm{E}$ and back $\mathrm{E}$ detectors was $140 \mu \mathrm{m}$ and $1000 \mu \mathrm{m}$ respectively. A beam of $45 \mathrm{MeV}{ }^{3} \mathrm{He}$ was used on a highly enriched ${ }^{157} \mathrm{Gd}$ target to produce compound nuclei of ${ }^{156} \mathrm{Gd}$ and ${ }^{157} \mathrm{Gd}$ through the ${ }^{157} \mathrm{Gd}\left({ }^{3} \mathrm{He}, \alpha\right){ }^{156} \mathrm{Gd}$ and ${ }^{157} \mathrm{Gd}\left({ }^{3} \mathrm{He},{ }^{3} \mathrm{He}\right)^{157} \mathrm{Gd}$ reactions. A total of 62 hours of particle- $\gamma$ and particle-singles data were recorded at an average current of 0.2-0.3 particle-nanoamperes. A full set of $\gamma$-ray and particle calibration sources were used for calibration.

Particle-identification was determined by sorting the off-line data into $\Delta \mathrm{E}$ - vs. $\mathrm{E}$ matrices. Figure 1 shows one of these matrices. The $\alpha-\gamma$ and ${ }^{3} \mathrm{He}-\gamma$ coincident data was then sorted into total energy vs. $\gamma$ ray matrices. The intensity of the most important $\gamma$-ray transitions in ${ }^{154-156} \mathrm{Gd}$ as a function of ejectile energy was determined by slicing on this matrix. A sum of non-coincident $\gamma$-rays, $\Gamma_{\mathrm{x}}\left(\mathrm{E}_{\mathrm{x}}\right)$, was then formed for the strongest transitions observed in the $\left({ }^{3} \mathrm{He}, \alpha \times \mathrm{xn}\right)$ and the $\left({ }^{3} \mathrm{He},{ }^{3} \mathrm{He} \mathrm{xn}\right)$ channels following the approach discussed in ref. [4]:

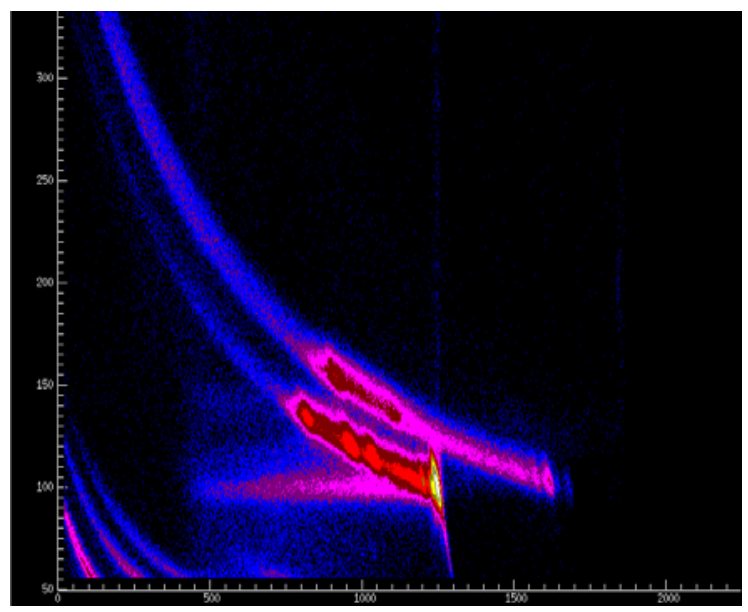

FIGURE 1. The $\Delta \mathrm{E}-\mathrm{E}$ matrix of the STARS+GAMMASPHERE experiment. The $\alpha$ and ${ }^{3} \mathrm{He}$ particles are clearly separated.

$$
\Gamma_{y}\left(E_{x}\right)=\sum_{i=\text { non-coincident }} I_{\gamma}^{i}\left({ }^{3} H e, \alpha y n \gamma_{i}\right)
$$

Where $I_{y}{ }^{l}$ are the efficiency and electron-conversion corrected intensities of the strongest non-coincidence 
$\gamma$-ray transitions $\gamma_{i}$. The ratio of the $\Gamma_{\mathrm{x}}\left(\mathrm{E}_{\mathrm{x}}\right)$ for a given channel was then divided by the sum over all open channels on order to determine the decay probability of the compound nucleus into that channel, i.e.:

$$
P_{y}\left(E_{x}\right)=\frac{\Gamma_{y}\left(E_{x}\right)}{\sum_{i=0}^{2} \Gamma_{i}\left(E_{x}\right)}
$$

\section{Reaction modeling}

We used the statistical model code STAPRE (STAatistical-PREequilibrium) to model the $\gamma$-ray decay cross sections for comparison to our data [5]. STAPRE includes a Hauser-Feshbach treatment of statistical decay and a simple exciton model of preequilibrium decay. STAPRE models the $\gamma$-ray decay of the residual nuclei using a statistical level density and $\gamma$-ray strength function at high energies that maps to the measured $\gamma$-ray cascade at a low enough energy where complete level spectroscopy is known.

The ratios from eq. [4] allow direct comparison between the decay of the nuclei formed using the ${ }^{3} \mathrm{He}$ induced direct reactions and neutron-induced reactions on different targets. Figure 2 shows a comparison between the ratios for the ${ }^{157} \mathrm{Gd}\left({ }^{3} \mathrm{He}, \alpha 2 \mathrm{n} \gamma\right){ }^{154} \mathrm{Gd}$ and the ${ }^{157} \mathrm{Gd}\left({ }^{3} \mathrm{He}, \alpha \gamma\right){ }^{156} \mathrm{Gd}$ channels from the STARS data and STAPRE calculations for the ${ }^{155} \mathrm{Gd}(\mathrm{n}, 2 \mathrm{n}){ }^{154} \mathrm{Gd}$ and ${ }^{155} \mathrm{Gd}(\mathrm{n}, \gamma){ }^{156} \mathrm{Gd}$ reactions.

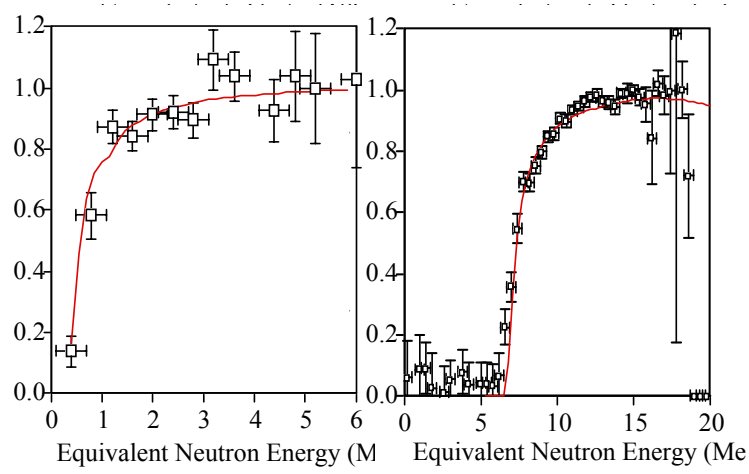

FIGURE 2. Decay probabilities for the $\left({ }^{3} \mathrm{He},{ }^{3} \mathrm{He} \mathrm{n} \gamma\right)$ (left) and the $\left({ }^{3} \mathrm{He}, \alpha y\right.$ (right) respectively from the STARS+GAMMASPHERE experiment (data points) compared to the same quantity from the STAPRE calculations for ${ }^{156} \mathrm{Gd}\left(\mathrm{n}, \mathrm{n}^{\prime} \gamma\right)$ and ${ }^{155} \mathrm{Gd}(\mathrm{n}, 2 \mathrm{n} \gamma)$ reactions. The STARS at Yale Experiment
The agreement between experiment and theory is remarkably good. It should be noted that the poor resolution of the particle data (FWHM=360 keV) make direct comparison between the data and the STAPRE calculations particularly difficult at low energies. Nonetheless, the agreement between the data and the model is particularly compelling at low energies where the angular momentum mismatch between the direct and the neutron-induced reactions are maximal. Similar agreement is seen for the ${ }^{157} \mathrm{Gd}\left({ }^{3} \mathrm{He},{ }^{3} \mathrm{He}\right.$ 'xn $\left.\gamma\right)$ STARS data and the ${ }^{156} \mathrm{Gd}(\mathrm{n}, \gamma)$ calculations using STAPRE.

\section{THE STARS AT YALE EXPERIMENTS}

The second series of experiments were carried out in April and May of 2004 at the A.W. Wright Nuclear Structure Laboratory at Yale University. Two "S2" annular double-sided Silicon detectors from Micron Semiconductor were used in STARS for this run. The S2 detectors consist of 48 rings on the ground plane and 16 sectors on the Junction side. However, adjacent rings and sectors were electrical connected producing a total of 24 azimuthal and 8 polar angles with respect to the beam. $24 \mathrm{MeV}$ and $32 \mathrm{MeV}$ deuteron beams were used on two Uranium Nitrate targets $\left({ }^{236} \mathrm{U}\right.$ and $\left.{ }^{238} \mathrm{U}\right)$ and a single Ammonium Nitrate target "stippled" on a $200 \mu \mathrm{g} / \mathrm{cm}^{2}$ Carbon backing. The140 $\mu \mathrm{m}$ thick front detector was used to as both the $\Delta \mathrm{E}$ component of the particle telescope and as a coincident fission fragment detector. Two $1000 \mu \mathrm{m}$ thick "back" detectors were used to provide additional stopping power for the Hydrogen isotope ejectiles. The front detector and the complete telescope covered an angular range of $36.2^{\circ}-66.8^{\circ}$ and $36.2^{\circ}-56.3^{\circ}$ each with respect to the beam. The YRAST ball (YaleRochester Array for Spectroscopy) array, consisting of 8 "clover" Ge detectors was used for $\gamma$-ray detection, but is not used in this analysis. Although the $\Delta \mathrm{E}$ separation between the isotopes of Hydrogen is not as clear as the ${ }^{3} \mathrm{He}$ and $\alpha$ ejectiles in the GAMMASPHERE + STARS data it is still sufficient to assure good particle identifications.

The ratio of the number of fission events in coincidence with deuterons for the ${ }^{236} U$ and the ${ }^{238} U$ targets was used to obtain the relative fission probability for the excited nuclei formed via (d,d') and $(\mathrm{d}, \mathrm{p})$ reactions in order to avoid the large systematics uncertainties arising from the Carbon, Nitrogen and Oxygen in the target. The ratios were corrected for the differing amount of target material (determined using the number of counts in the elastically scattered 
peaks), different running times (determined by the master trigger rate), and at low energy for the different particle-fission fragment angular correlation for the two different compound nuclei. The ratios of fission probability are inherently more robust than the fission probability since systematics uncertainties in the detector efficiencies and the particle singles spectrum cancel out. However, in comparing these ratios to the ratios for neutron-induced reactions the assumption is made that the direct reactions on the ${ }^{236} \mathrm{U}$ and the ${ }^{238} \mathrm{U}$ targets are essentially identical.

Figures 4 above shows the ${ }^{236} \mathrm{U}(\mathrm{d}, \mathrm{pf}) /{ }^{238} \mathrm{U}(\mathrm{d}, \mathrm{pf})$ and the $\left.{ }^{238} U\left(d, d^{\prime} f\right)\right)^{236} U\left(d, d^{\prime} f\right)$ ratios respectively. Detector "punch through" and the Coulomb barrier of the ejectiles determine the energy limits of the meausrement. The good agreement between the measured ${ }^{236} \mathrm{U}(\mathrm{d}, \mathrm{pf}) /{ }^{238} \mathrm{U}(\mathrm{d}, \mathrm{pf})$ ratio and the ${ }^{236} \mathrm{U}(\mathrm{n}, \mathrm{f}){ }^{238} \mathrm{U}(\mathrm{n}, \mathrm{f})$ from ENDF-B6 lends confidence in using equating the ${ }^{238} \mathrm{U}\left(\mathrm{d}, \mathrm{d}^{\prime} \mathrm{f}\right){ }^{236} \mathrm{U}\left(\mathrm{d}, \mathrm{d}^{\prime} \mathrm{f}\right)$ ratio to the ${ }^{237} \mathrm{U}(\mathrm{n}, \mathrm{f}){ }^{235} \mathrm{U}(\mathrm{n}, \mathrm{f})$ ratio. Also shown in Figure 4 are the ratio of the predictions of Younes [7] for the ${ }^{237} \mathrm{U}(\mathrm{n}, \mathrm{f})$ over the ${ }^{235} \mathrm{U}(\mathrm{n}, \mathrm{f})$ cross section from ENDFB6. The agreement between the (d,d'f) ratios and the prediction of Younes is remarkably good over the energy range covered in these experiment.

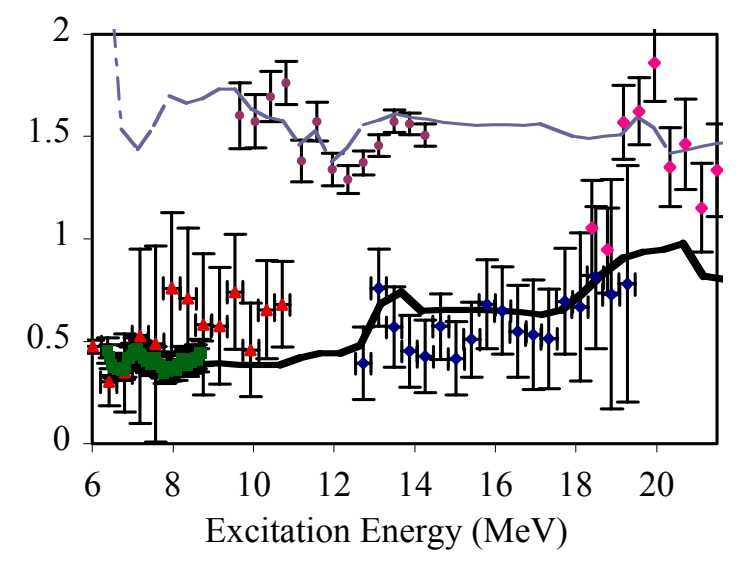

FIGURE 3. Fission probability ratios for STARS for 24 (red and purple points) and $32 \mathrm{MeV}$ (pink and blue points) deuteron beam energies. The lower two ratios are for ${ }^{238} \mathrm{U}(\mathrm{d}, \mathrm{d}$ 'f $){ }^{236} \mathrm{U}(\mathrm{d}, \mathrm{d}$ 'f $)$. The solid curve and the green data points are from [7] and [3] respectively. The upper two ratios are for ${ }^{236} \mathrm{U}(\mathrm{d}, \mathrm{pf}) /{ }^{238} \mathrm{U}(\mathrm{d}, \mathrm{pf})$. The top curve is the ratio of ${ }^{236} \mathrm{U}(\mathrm{n}, \mathrm{f}) /{ }^{238} \mathrm{U}(\mathrm{n}, \mathrm{f})$ from ENDF-B6.

\section{CONCLUSIONS}

The results of two surrogate reactions measurements have been presented. In the first case, excellent agreement is observed between neutron decay probabilities inferred from discrete $\gamma$-ray yields for ${ }^{3} \mathrm{He}$-induced reactions and statistical model calculations of neutron-induced reactions leading to the same intermediate nucleus. In the second case, the ${ }^{236} \mathrm{U}(\mathrm{d}, \mathrm{pf}){ }^{238} \mathrm{U}(\mathrm{d}, \mathrm{pf})$ ratio matched the evaluated ${ }^{236} \mathrm{U}(\mathrm{n}, \mathrm{f}){ }^{238} \mathrm{U}(\mathrm{n}, \mathrm{f})$ ratio over a wide range of energy. We have then used the ${ }^{238} \mathrm{U}\left(\mathrm{d}, \mathrm{d}^{\prime} \mathrm{f}\right){ }^{236} \mathrm{U}\left(\mathrm{d}, \mathrm{d}^{\prime} \mathrm{f}\right)$ ratio to deduce the ${ }^{237} \mathrm{U}(\mathrm{n}, \mathrm{f}) \mathrm{P}^{235} \mathrm{U}(\mathrm{n}, \mathrm{f})$ fission cross section over an unprecedented range of surrogate energy. These results indicate that the that the surrogate reaction technique holds significant promise as a tool to deduce neutron-induced cross sections for reactions that are difficult to quantitatively predict or measure. However, additional theoretical and experimental work is needed to determine the limits of the surrogate reaction approach.

\section{ACKNOWLEDGMENTS}

This work was performed under the auspices of the U.S. Department of Energy by the University of California, Lawrence Livermore National Laboratory under Contract No. W-7405-Eng-48, Lawrence Berkeley National Laboratory under contract No. DEAC03-76SF0098 (LBNL), and by the U.S. Department of Energy Grant Nos. DE-FG02-91-ER40609, DEFG03-03NA-00081 (Yale) and the Yale University Flint fund.

\section{REFERENCES}

1. H.C. Britt et al., Phys. Rev. 1751525 (1968).

2. W.Younes, H.C.Britt, Phys.Rev. C67, 024610 (2003)

3. W. Younes, H.C.Britt Phys.Rev. C68, 034610 (2003)

4. L.A. Bernstein et al., Phys. Rev C65 021601R (2002).

5. W. Hauser and H. Feshbach, Phys. Rev. 87, 366 (1952).

6. M. Uhl, B. Strohmaier, IRK-Vienna Report IRK-76/01 1976 (Upd. 1978).

7. W. Younes et al., Initial Estimate for the ${ }^{237} \mathrm{U}(\mathrm{n}, \mathrm{f})$ cross section for $0.1<\mathrm{E}_{\mathrm{n}}(\mathrm{MeV})<20$ UCRL-ID-154194. (2003). 